\title{
Elternstatus, Lehrerempfehlung und Schullaufbahn: Eine empirische Analyse des Einflusses des Grundschullehrers auf die Bildungslaufbahn des Schülers
}

\section{Wilhelm Wiese}

Universität zu Köln, Zentralarchiv für empirische Sozialforschung

Bachemer Straße 40, D-5000 Köln 41

Z u s a m m e n a s s u n g: Der Einfluß der Empfehlung des Primarschullehrers auf die weitere Schullaufbahn des Kindes wird untersucht. Die schulischen Präferenzen der Eltern und die Empfehlungen der Lehrer bestimmen die Bildungsentscheidung am Ende des 4. Schuljahrs Grundschule. Zunächst werden Hypothesen zum Einfluß von Elternstatus und Schulleistung des Kindes auf Schulpräferenzen der Eltern und Empfehlungen der Lehrer und zum Einfluß der Lehrerempfehlung auf die Bildungsentscheidung abgeleitet. Die Hypothesen werden in einem Kausalmodell zusammengefaßt und das Modell durch eine Pfadanalyse getestet. Es zeigt sich ein unerwartet starker Einfluß der Lehrerempfehlung auf die Bildungsentscheidung. Die analytische Trennung von direkten und über die Schulleistung des Kindes vermittelten Schichteffekten auf die Lehrerempfehlung führt zu Erklärungen, die von bisherigen Ergebnissen abweichen.

\section{Einleitung}

Die Bedeutung von Bildungsabschlüssen für den späteren beruflichen Werdegang und die Ubertragung des elterlichen Status auf das Kind ist durch zahlreiche makrosoziologische Untersuchungen belegt. Während die schichtenspezifischen Einflußfaktoren des Elternhauses auf die Bildungslaufbahn des Kindes relativ häufig untersucht werden, gibt es nur wenige Untersuchungen, die sich mit dem Einfluß der Empfehlung des Grundschullehrers auf die Bildungslaufbahn des Kindes beschäftigen (Steinkamp 1967; Preuß 1968; Gresser-Spitzmüller 1973). In einem dreiglied rigen Schulsystem müssen relativ früh Entscheidungen über die weitere Schullaufbahn des Kindes getroffen werden, die nur langfristig revidierbar sind. Neben den eigenen Wünschen und Zielen können sich die Eltern mehr oder weniger an den schulischen Noten des Kindes und der Empfehlung des Grundschullehrers orientieren. Die meisten Untersuchungen beschränken sich darauf, entweder schichtenspezifische Einflüsse des Elternstatus oder der Lehrer auf die Bildungslaufbahn nachzuweisen. Wird der Einfluß der Schichtzugehörigkeit des Schülers auf die Lehrerempfehlung aufgedeckt, so wird allzu schnell nach Gründen und Erklärungen für diesen Zusammenhang gesucht, ohne das Gewicht des Einflusses im Zusammenwirken mit anderen wichtigen Einflußgrößen der Bildungsentscheidung festzustellen. Ziel dieser Sekundäranalyse ist es, die Einfluisse von Elternhaus (schulische Ziele und Wünsche der Eltern) und Schule (Leistungsstand des Schülers/Empfehlung des Grundschullehrers) gegenüberzustellen und zu vergleichen.

\section{Fragestellungen und Hypothesen}

Im ersten Teil der Arbeit sollen Fragestellungen und Hypothesen zu den relevanten EinflußgröBen der Bildungsentscheidung formuliert werden. Dabei werden Teile eines komplexeren Wirkungszusammenhangs zunächst isoliert behandelt und später in einem Gesamtmodell zusammengefaßt. Im zweiten Teil der Arbeit werden die Hypothesen mit den Ergebnissen einer multivariaten Kausalanalyse verglichen.

\subsection{Statusinteressen der Eltern und Bildungs- wünsche für das Kind}

Eltern aller Sozialschichten werden für ihre Kinder zumindest die Bildungsabschlüsse vorsehen, die den Statuserhalt sichern. Entsprechend der sozialen Lage der Eltern ergeben sich unterschiedliche schulische Aspirationen für die Kinder. Statuserhalt bedeutet für Angehörige höherer Schichten zumeist, daß die Kinder das Bildungsziel Abitur erreichen müssen, um nicht abzusteigen; Angehörige unterer Sozialschichten können den Status durch einfachere Bildungsabschlüsse erhalten. Allerdings können Bildungspläne der Eltern von den weitgehend als selbst verständlich angesehenen Bildungszielen abweichen. Breitere Bildungsangebote, Bildungswerbung, Veränderungen traditioneller Gesellschaftsbilder, Aufstiegswille usw. verändern Bildungsperspektiven, die früher mit der sozialen Lage verbunden waren. Der Bildungsaufstieg ist - neben den unteren Schichten - vor allem für Angehörige mittlerer Schichten möglich. Mittelschichteltern sind gegenüber Unterschichteltern 
in der Planung und Realisierung schulischer Laufbahnen im Vorteil, weil mit ihrer Position in der Sozialstruktur weniger starre Bildungsperspektiven verbunden sind und Vor- und Nachteile, Chancen und Risiken höherer Bildungsgänge eher überschaut werden. Je nach sozialer Ausgangslage werden Eltern also mehr oder weniger weite Bildungsperspektiven haben, entsprechend den schulischen Bildungsweg für ihre Kinder planen und ihre Bildungsansprüche an die Schule herantragen. Die Schule setzt den individuellen Bildungsansprüchen der Eltern gesellschaftliche Leistungsnormen entgegen. Die Leistungsfähigkeit der Schüler wird regelmäßig durch Schulnoten und Zeugnisse dokumentiert, darüber hinaus macht die Schule ihren Einfluß am Ende des 4. Grundschuljahres beim Ubergang auf die weiterführenden Schulen durch eine Empfehlung des Grundschullehrers geltend.

Schulische Leistungskriterien und Bildungsansprüche der Eltern stehen während der Grundschulzeit in einem Spannungs- und Beeinflussungsverhältnis. In der Regel werden hohe schulische Aspirationen der Eltern, die häufiger in höheren Schichten zu finden sind, auch zu höheren Leistungen des Kindes führen - einmal weil von vornherein ein höheres Anspruchsniveau gesetzt und auf dessen Einhaltung geachtet wird, zum anderen weil mit höheren Aspirationen häufig ein höherer intellektueller und kultureller Anregungsgehalt des Elternhauses verbunden ist. Auf der anderen Seite können schulische Leistungsbescheide hohe schulische Ziele der Eltern durchkreuzen oder auch ursprünglich niedrige schulische Aspirationen der Eltern verändern. Zum Zeitpunkt der Ubergangsentscheidung am Ende des 4. Schuljahres haben Eltern und Kinder eine vierjährige schulische Erfahrungszeit hinter sich, in der sich derartige Einfliusse und Veränderungen vollzogen haben können.

Den schulischen Zielen und Wünschen der Eltern steht nun die mehr oder minder erfolgreiche und kontinuierliche schulische Leistungslaufbahn des Kindes gegenüber. Welche Bildungsziele und -ansprüche werden die Eltern zu diesem Zeitpunkt haben, unbeeinflußt von der Empfehlung des Grundschullehrers? Welche Beziehung wird zwischen der Bildungspräferenz der Eltern und den Schulleistungen des Kindes bestehen? Je höher die Schichtzugehörigkeit der Eltern, um so höher werden die schulischen Aspirationen sein, um so höher wird aber auch die schulische Leistungsfähigkeit des Kindes sein. Verglichen mit der Gesamtheit der Eltern und gemessen an den schulischen Leistungen des Kindes sind die schulischen Aspirationen von Unterschichteltern häufig niedriger und die der Oberschichteltern häufig höher. Da ein stärkerer Zusammenhang zwischen der Schichtzugehörigkeit der Eltern und den schulischen Aspirationen als zwischen Schichtzugehörigkeit der Eltern und den schulischen Leistungen der Kinder vermutet wird, wird es vor allem in den oberen und unteren Schichten zu Diskrepanzen zwischen schulischem Leistungsniveau der Kinder und Bildungsansprüchen der Eltern kommen. Während in der Mittelschicht häufig mittlere Bildungsansprüche der Eltern mit durchschnittlichen Leistungen der Kinder zusammentreffen, liegen die Bildungsansprüche von Unterschichteltern häufig unter und die Bildungsansprüche von Oberschichteltern häufig über dem Leistungsniveau der Kinder.

Die Position des Elternhauses in der Sozialstruktur und der Wunsch der Eltern, den eigenen Status auf die Kinder zu übertragen, führen so zu entsprechenden Diskrepanzen zwischen dem schulischen Leistungsniveau des Kindes und dem Aspirationsniveau der Eltern. Wie stark werden sich die Eltern an ihren Statusinteressen und an den schulischen Leistungen des Kindes orientieren? Wie stark wird der Schichteinfluß auf die Schulpräferenzen der Eltern sein und inwieweit wird der Schichteffekt über die schulische Leistungsfähigkeit der Kinder vermittelt?

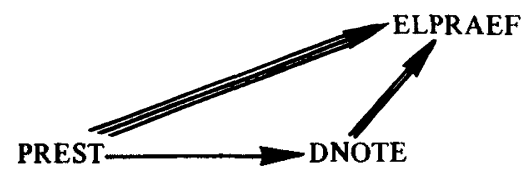

ABBILDUNG 1 Teilmodell Elternpräferenz

In Abbildung 1 sind die Beziehungen zwischen der Schichtzugehörigkeit der Eltern (Prestige $=$ PREST) ${ }^{1}$, schulischem Leistungsniveau des Kindes (Durchschnittsnote $=$ DNOTE) und schulischen Präferenzen der Eltern (Elternpräferenz $=$ ELPRAEF) dargestellt. Die oben gestellten Fragen sol-

1 Zur Zuweisung von Prestigewerten zu den Berufsangaben siehe Abschnitt 3: Daten und Variablen der Sekundäranalyse. 
len hypothetisch soweit beantwortet werden, als Aussagen über die vergleichsweise stärkeren und schwächeren Kausaleinflüsse gemacht werden, ohne diese jedoch zu quantifizieren. Aus den statusbedingten Interessen und Ansprüchen der Eltern läßt sich der dominante Kausaleinfluß ableiten. Die schulischen Präferenzen der Eltern werden stärker status- als leistungsbezogen sein. Der indirekte Schichteffekt auf die Schulpräferenzen der Eltern, der durch höhere schulische Leistungen der Kinder zustandekommt, wird geringer sein als der direkte Schichteffekt auf die Schulpräferenz der Eltern.

\subsection{Leistungsnormen der Schule und Empfehlungen der Lehrer}

Während die schichtenspezifischen Einflüsse auf die Bildungslaufbahn des Kindes auf seiten des Elternhauses durch Ziele und Interessen bestimmt werden können, lassen sich Vermutungen über schichtenspezifische Einfliusse auf seiten der Schule weniger eindeutig ableiten.

Schichtenspezifische Einflüsse der Schule können sich einmal auf die Leistungen des Kindes auswirken, zum anderen auf die schulischen Pläne und Entscheidungen der Eltern. Bezogen auf die Leistungen des Kindes hat die Schule, je nachdem welche gesellschaftspolitische Zielsetzung unterstellt wird, verschiedene Aufgaben. Einmal soll sie Nachteile und ungleiche Startchancen von Kindern, die mit der sozialen Herkunft zusammenhängen, ausgleichen, zum anderen soll sie für eine leistungsgerechte Beurteilung des Schülers und leistungsgerechte Selektion sorgen. Unter idealen Annahmen besteht zwischen diesen Aufgaben kein Widerspruch. Gelänge es der Schule, schichtenspezifische Nachteile auszugleichen, so wären Schüler aller Schichten in den Leistungsgruppen anteilig repräsentiert. Zwischen leistungsgerechter Beurteilung und Repräsentation in den Leistungsgruppen bestïnde keine Diskrepanz. Tatsächlich zeigen sich aber in den Leistungen der Kinder erste schichtenspezifische Einfluisse, deren Ursachen man im Elternhaus oder in der Schule suchen kann. Der Schule wird in diesem Zusammenhang vorgeworfen, daß sie leistungsfremde Aspekte bei der Beurteilung von Schülern berücksichtigt und mit der sozialen Herkunft verbundene Nachteile von Schülern nicht kompensiert. Diese Schichteinflüsse auf die Lei- stungen des Kindes können hier nicht untersucht werden, da keine Schulleistungs- und Intelligenztests zur Verfügung stehen. Unter den gegebenen Untersuchungsbedingungen müssen die Schulnoten der Kinder als Definition von Begabung und Leistung durch die Schule selbst hingenommen werden.

Ein unmittelbarer Einfluß auf die Schullaufbahn des Schülers besteht aber in der Empfehlung des Grundschullehrers für eine der weiterführenden Schulen am Ende des 4. Schuljahres. Wird die Empfehlung des Lehrers sich ausschließlich an schulischen Leistungskriterien orientieren - werden also die Noten des Schülers Grundlage der Empfehlung sein - oder wird sie auch von der Schichtzugehörigkeit des Schülers beeinflußt sein? Zunächst lassen sich keine unmittelbaren Interessen der Lehrer bestimmen, die vergleichbar wären mit den Statusinteressen der Eltern und für stark schichtenbezogene Empfehlungen sprechen würden. Schichteinflüsse auf die Lehrerempfehlung können aber auf verschiedene Weise zustandekommen: einmal können Eltern höherer Schichten mit hohen schulischen Aspirationen häufiger Gelegenheiten zum Gespräch mit dem Lehrer nutzen, um Einfluß auf die Leistungsurteile des Lehrers und damit auch auf die Leistungslaufbahn des Kindes zu nehmen. Die pädagogische Mitarbeit der Eltern kann vom Lehrer als günstige Bedingung für eine höhere Schullaufbahn beurteilt werden. Weiter können Lehrer neben der schulischen Leistung des Kindes zusätzliche Kriterien zu Beurteilung des Schülers heranziehen. Wenn die Vorstellungen des Lehrers vom ,guten" Schüler eher mit Begabungs- und Verhaltensmerkmalen von Schülern höherer Schichtzugehörigkeit übereinstimmen, wird es ebenfalls zu einem Schichteinfluß auf die Lehrerempfehlung kommen. Schließlich ist denkbar, daß der Lehrer die Empfehlung als Prognose über Erfolg oder Mißerfolg des Schülers in der weiteren Schullaufbahn auffaßt. Er wird also neben den Leistungen des Schülers Bedingungen des Elternhauses berücksichtigen. Auch bei guten schulischen Leistungen des Schülers könnte er sich für die Empfehlung einer niedrigen Schullaufbahn entscheiden, wenn er Bedenken wegen mangelhafter Unterstützung auf seiten des Elternhauses hat.

In Abbildung 2 sind die vermuteten Beziehungen zwischen dem Elternstatus, den Schulleistungen 


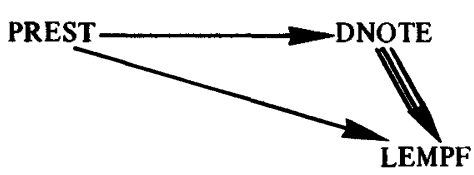

ABBILDUNG 2 Teilmodell Lehrerempfehlung

des Kindes und der Lehrerempfehlung (LEMPF) dargestellt. Obwohl das Teilmodell „Lehrerempfehlung" formal und hinsichtlich der unabhängigen Variablen dem Teilmodell „Elternpräferenz“ gleich ist, unterscheiden sich die Modelle grundsätzlich in der Kausalstruktur und den Handlungsprinzipien, die zur abhängigen Variablen, den Vorstellungen über die schulische Zukunft des Kindes führen. Für die Eltern geht es darum, ein statusgemäßes Bildungsziel für das Kind zu erreichen; die Schulleistung des Kindes ist dabei eine mehr oder minder ,störende“ Bedingung der schulischen Lebensplanung. Die Lehrer dagegen haben kein unmittelbares Interesse an einer statusbezogenen Auslese, für sie sind die Schulleistungen der Kinder von zentraler Bedeutung. Leistungen von Schülern zu messen, zu quantifizieren und zwischen Schülern zu vergleichen, gehört zur täglichen Arbeit des Lehrers. Handeln und Verhalten des Schülers wird durch die ,gemessene" Leistung bewertet und belohnt, Schulnoten bestimmen seinen Platz in der Leistungshierarchie der Klasse. Auch wenn die Leistungsmessung in einigen Fächern umstritten ist und eine objektive Notengebung de facto nicht immer erreicht wird, so gehört eine möglichst objektive Beurteilung der Schülerleistung zum normativen Selbstverständnis des Lehrerberufs; das Leistungsprinzip hat als allgemeines Handlungsprinzip des Lehrers Gültigkeit. Für die Übergangsempfehlung des Lehrers sind die Schulnoten des Kindes deshalb die wichtigste Orientierungsgröße. Trotzdem wird die Lehrerempfehlung keine „mechanistische" Umsetzung der Schulnoten in einen entsprechenden Rat an die Eltern darstellen. Der Lehrer wird seine Empfehlung vielmehr als eine Art Prognose über die Erfolgsmöglichkeiten des Schülers verstehen. Seine Handlungsmöglichkeiten werden dabei zunächst durch die Schulleistung des Schülers bestimmt. Darüberhinaus aber wird er sich in die Lage des Schülers versetzen, den familiären Hintergrund und das soziale Umfeld der Eltern bedenken und stellvertretend für Schüler und Eltern aufgrund seiner beruflichen Kompetenz und Erfahrung ein Bildungsziel bestimmen. Da sich Chancen und Risiken, Mög- lichkeiten des Erfolgs oder Mißerfolgs mit der so. zialen Herkunft des Kindes verändern, kann es bei Schülern mit gleich guten Schulleistungen zu unterschiedlichen Empfehlungen des Lehrers kommen. Die mit der sozialen Herkunft des Schülers verbundenen Bedingungen werden aber im Vergleich zu den Schulleistungen geringe Bedeutung haben.

Da der Lehrer seine Empfehlung nicht ausschließlich an schulischen Leistungskriterien orientiert, kommt es zu einem direkten Schichteffekt. Ein indirekter Schichteffekt entsteht dadurch, daß die Schulleistung durch die Schichtzugehörigkeit des Kindes beeinflußt ist und der Lehrer seine Empfehlung weitgehend an schulischen Leistungskriterien orientiert. Auch wenn die Empfehlung des Lehrers sich ausschließlich an schulischen Leistungskriterien orientieren würde, käme es zu einem indirekten Schichteffekt.

Welcher der Effekte wird stärker sein, der direkte oder der indirekte Schichteffekt? Da nur ein schwacher Schichteinfluß auf die Schulleistungen der Kinder angenommen wird und die Übertragung dieses Schichteffekts auf die Lehrerempfehlung von der Leistungsbezogenheit der Lehrerempfehlung abhängt, wird der direkte Schichteffekt stärker als der indirekte Schichteffekt sein. Insgesamt werden die Schichteffekte auf die Lehrerempfehlung geringer sein als die Schichteffekte auf die Elternpräferenz.

\subsection{Der Einfluß der Lehrerempfehlung auf die Bildungsentscheidung der Eltern}

Nachdem die vermuteten Zusammenhänge zwischen Elternstatus, Schulleistung des Kindes, Elternpräferenz und Lehrerempfehlung dargelegt worden sind, soll die abhängige Variable, die Bildungsentscheidung der Eltern am Ende des 4. Schuljahres, behandelt werden:

Die Bildungsentscheidung (Schulwahl $=$ SCHULW) wird als Ergebnis der schulischen Präferenzen der

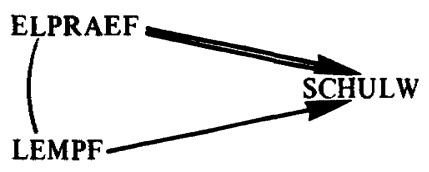

ABBILDUNG 3 Teilmodell Bildungsentscheidung 
Eltern (ELPRAEF) und der Empfehlungen der Lehrer (LEMPF) angesehen. Eltern und Lehrer beurteilen die vergangene und planen die zukünftige Bildungslaufbahn des Schülers nach unterschiedlichen Prinzipien. Die individuellen Statusinteressen der Eltern führen zu Bildungswünschen für das Kind, die allgemeinen Leistungsnormen der Schule zu Eignungs- und Erfolgsprognosen der Lehrer über den weiteren schulischen Lebensweg des Kindes. Trotz unterschiedlicher Handlungsprinzipien von Eltern und Lehrern wird eine starke Beziehung zwischen den Bildungspräferenzen der Eltern und den Empfehlungen der Lehrer bestehen. In vielen Fällen werden Eltern und Lehrer die gleichen Bildungsvorstellungen für das Kind haben, weil keine einseitig status- bzw. leistungsbezogenen Bildungsvorstellungen vorliegen ${ }^{2}$. Trotz dieser starken Beziehung wird ein kausaler Einfluß der Lehrerempfehlung auf die Elternpräferenz - und umgekehrt - im vorliegenden Modell ausgeschlossen. Die Kausalanalyse bezieht sich auf die Ubergangssituation am Ende des 4. Schuljahres.

Für die Zeitpunktbetrachtung ist wesentlich, daß unterschiedliche Handlungsprinzipien und Interessen von Eltern und Lehrern zu den Bildungsvorstellungen geführt haben. Keine der beiden Variablen ist in dem Sinne zeitlich vor- oder nachgeordnet, daß eine einseitige oder wechselseitige Beeinflussung möglich wäre. Möglichkeiten des Einflusses auf die Leistungen des Kindes und damit auch indirekt auf die Empfehlung des

2 Mit der Lehrerempfehlung (LEMPF) und der Elternpräferenz (ELPRAEF) befinden sich zwei Prediktorvariablen in dem Kausalmodell, die stark miteinander korrelieren. Redundanz und Repetition von Variablen werden in der statistischen Literatur zur Pfadanaly se als Probleme der Multikollinearität behandelt. Redundanz liegt vor, wenn in verschiedenen Variablen inhaltlich gleiche Informationen enthalten sind. Repetition bezieht sich auf die Anzahl von Variablen mit gleichen Informationen. Der Effekt von Multikollinearität besteht darin, daß der gemeinsame prognostische Wert der Variablen kaum zunimmt, sondern auf verschiedene Variablen verteilt wird, was zu einem Sinken der absoluten Werte der Koeffizienten für die einzelnen Variablen fihrt. Diese Folge von Multikollinearität sind z.B. bei Indikatorvariablen zum sozioökonomischen Status unerwünscht. Außerdem können Vorzeichenwechsel und Koeffizienten, die größer als 1 sind, auftreten (Gordon 1968: 592-616). Im vorliegenden Modell ist aber gerade die Aufteilung der Korrelationen zwischen den beiden Variablen das Ziel der Analyse, Multikollinearität aus theoretischen Gründen also unvermeidbar.
Lehrers sind von den Eltern schon während der Grundschulzeit wahrgenommen worden. Vor allem Eltern mit hohen schulischen Aspirationen werden besonders auf die schulischen Leistungen des Kindes achten und versuchen, über gute Kontakte zum Lehrer mehr oder minder direkt dessen Leistungsurteile zu beeinflussen. Diese Einflüsse sind im Kausalmodell 2 durch den Pfad von Elternstatus (PREST) zur Lehrerempfehlung (LEMPF) berücksichtigt.

Wie wird die endgültige Bildungsentscheidung der Eltern ausfallen? Beeinflußt die Empfehlung des Lehrers die Bildungsentscheidung der Eltern oder bleiben die Präferenzen der Eltern weitgehend unverändert? Die Einschätzung der Einflußstärke von Elternpräferenz und Lehrerempfehlung muß zugunsten der Elternpräferenz ausfallen. Einmal ist die Empfehlung des Grundschullehrers für die Eltern unverbindlich, zum anderen kann angenommen werden, daß die Eltern ihre statusbedingten Interessen durchsetzen wollen. Ebenso wie die Bildungspräferenzen der Eltern als in geringerem Maße leistungsbezogen angesehen wurden, wird die Bildungsentscheidung der Eltern weniger an den Lehrerempfehlungen orientiert sein und stärker an den eigenen Statusinteressen.

\subsection{Schicht- und Leistungseffekte auf die Bildungslaufbahn}

Die einzelnen Teilmodelle können jetzt zusammengefaßt werden und die Kausaleinflüsse des Gesamtmodells hinsichtlich der Fragestellungen und Hypothesen überprüft werden:

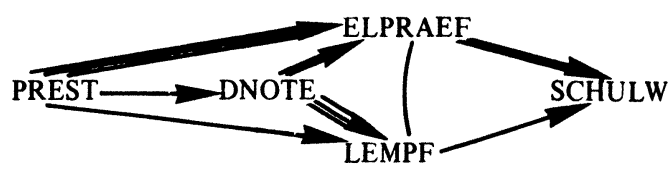

ABBILDUNG 4 Gesamtmodell

Die Bildungspräferenzen der Eltern wurden als stark statusbezogen, die Empfehlungen der Lehrer als stark leistungsbezogen angenommen. Entsprechend wurde ein stärkerer direkter Schichteffekt auf die Elternpräferenzen angenommen und ein stärkerer indirekter über die Schulleistung des Kindes vermittelter Schichteffekt auf die Lehrerempfehlung. Der gesamte Schichteffekt auf die Lehrerempfehlung müßte nach den 
Annahmen leistungsbezogener Lehrerempfehlungen und statusbezogener Bildungspräferenzen der Eltern schwächer sein als der gesamte Schichteffekt auf die Elternpräferenz. Die Frage nach der Stärke des Einflusses von Elternpräferenz und Lehrerempfehlung auf die Bildungsentscheidung wurde zugunsten der Bildungspräferenzen der Eltern entschieden. Das Gesamtmodell berücksichtigt alle bis jetzt theoretisch begründeten Kausaleinflüsse. Darüberhinaus sind aber Kausaleinflüsse im Gesamtmodell denkbar, die noch nicht behandelt worden sind.

Eine vollständige Aufteilung der Korrelation zwischen Elternstatus (PREST) und Bildungsentscheidung (SCHULW) auf vermittelnde Variablen wird nur dann gelingen, wenn alle wichtigen Einflußgrößen der Bildungsentscheidung erfaßt worden sind. Ist eine theoretisch wichtige unabhängige Variable, die mit dem Elternstatus und der Bildungsentscheidung zusammenhängt, nicht im Modell enthalten, so wird sich statistisch ein direkter kausaler Einfluß zwischen Elternstatus (PREST) und Bildungsentscheidung (SCHULW) ergeben. Neben der Elternpräferenz und der Lehrerempfehlung könnte der Schulwunsch des Kindes Einfluß auf die Bildungsentscheidung haben. Gegen einen eigenständigen Einfluß der schulischen Wïnsche der Kinder spricht aber einmal, daß die Präferenzen der Eltern nicht völlig unabhängig von den Wünschen der Kinder sind, zum anderen werden die Eltern primär den schulischen Lebensweg des Kindes bestimmen, da diese am Ende des 4. Schuljahres kaum älter als 10 Jahre sind. Das Kausalmodell soll also durch keine weitere Variable ergänzt werden. Weiter könnte ein direkter Pfad zwischen den Schulleistungen des Kindes (DNOTE) und der Bildungsentscheidung (SCHULW) bestehen. Ein eigenständiger direkter Einfluß der Schulleistung auf die Bildungsentscheidung erscheint aber nicht plausibel, weil die Lehrerempfehlung als stark leistungsbezogen angenommen wurde und damit Leistungseffekte auf die Bildungsentscheidung vermittelt werden. Außerdem wurde angenommen, daß die Bildungsentscheidung stark von den Bildungspräferenzen der Eltem bestimmt wird, so daB eine theoretische Begründung eines direkten Leistungseffekts auf die Bildungsentscheidung auch insofern schwerfallt. Das vorliegende Kausalmodell soll also nicht verändert werden. Es wird angenommen, daß die wichtigen Einflußgrößen und Pfade in der Kausalstruk- tur enthalten sind und weitere Einflüsse nicht begründet $\operatorname{sind}^{3}$. Die theoretisch angenommene Kausalstruktur wird später anhand der empirischen Ergebnisse getestet.

Welche Konsequenzen ergeben sich im Gesamtmodell im Hinblick auf die in den Einzelmodellen formulierten Hypothesen für die Ubertragung von Schicht und Leistungseffekten auf die Bildungsentscheidung? Welchen Einfluß werden die Unterschiede in der Leistungs- und Schichtbezogenheit von Elternpräferenz und Lehrerempfehlung auf das Ausmaß sozial ungleicher Bildungslaufbahnen der Schüler haben? Da die Lehrerempfehlung im Vergleich zur Elternpräferenz als stärker leistungsbezogen angenommen wurde, könnte von der Lehrerempfehlung ein nivellierender Einfluß auf die soziale Auslese am Ende des 4. Schuljahres ausgehen. Da aber nur ein schwacher Einfluß der Lehrerempfehlung auf die Bildungsentscheidung vermutet wird, werden sich nur schwache Leistungseffekte und starke Schichteffekte auf die Bildungsentscheidung auswirken. Bevor das Modell mit den empirischen Ergebnissen der multivariaten Kausalanalyse konfrontiert wird, sollen die Daten und Variablen der Sekundäranalyse vorgestellt werden.

\section{Daten und Variablen der Sekundäranalyse}

Die Untersuchung, die zur Sekundäranalyse herangezogen wurde, ist 1968 von der Arbeitsgruppe für empirische Bildungsforschung, Heidelberg, durchgeführt worden. Befragt wurden 1.279 Eltern und Lehrer von Schülern, die im Herbst 1967 in Baden-Württemberg den Uber-

3 Ein alternatives Modell, das formal gleichwertig bis auf den Pfad PREST-DNOTE ist, aber im Gegensatz zum vorliegenden Modell - in dem die zeitliche Abfolge der Kausaleinflüsse im Vordergrund steht - die Variablen nach der Zugehörigkeit zu den Sozialisationsagenturen Elternhaus und Schule ordnet, sieht wie folgt aus:

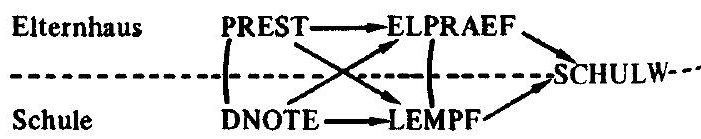

In diesem Modell wird der Einfluß der Schichtzugehörigkeit auf die Schulleistung des Kindes nicht kausal interpretiert. Ich danke Dr. Heiner Meulemann (Köln) für seine Hilfe bei der Aufstellung und Analy se von Kausalmodellen. 
gang von der 4. Klasse Grundschule in die 5. Klasse von Haupt- und Realschule oder Gymnasium vollzogen hatten. Aus der Befragung von Eltern und Lehrern ${ }^{4}$ wurde eine Gesamtdatei für die Schüler erstellt, zu denen sowohl ein Elternwie auch ein Lehrerinterview vorlag. Es handelt sich um eine doppelt geschichtete Auswahl nach drei Schultypen und sechs Berufsstellungsgruppen.

Für die Sekundäranalyse wird ein Klassenlagenkonzept verwandt (Meulemann 1977). Die Klassenlage ist definiert durch Dimensionen der beruflichen Stellung des Vaters (Arbeiter, Angestellte, Beamte, Selbständige), durch Abhängigkeit/Selbständigkeit von Berufen, durch manuelle und nichtmanuelle Arbeit und durch die berufliche Qualifikation. Den Berufsstellungsgruppen bzw. den Klassenlagen wurden die Berufe und Prestige-Scores zugeordnet, die Kleining und Moore nach dem Verfahren der sozialen Selbsteinstufung für 70 Berufe ermittelt haben (Kleining und Moore 1968). Die Prestige-Scores der zugeordneten Berufe wurden dann für jede Berufsstellungsgruppe gemittelt $(\text { Prestige }=\text { PREST })^{5}$.

\section{Die Variablen Bildungspräferenz der Eltern (EL-} PRAEF), Empfehlung des Grundschullehrers (LEMPF) und die Bildungsentscheidung (Schulwahl $=$ SCHULW) haben die Ausprägungen Hauptschule, Realschule und Gymnasium. Für die Pfadanalyse wurde den Kategorien die Dauer der Ausbildung zugeordnet, also 8 Jahre für den Hauptschulabschluß, $10 \mathrm{Jahre}$ für die mittlere Reife und 13 Jahre für das Abitur.

Als schulisches Leistungskriterium wurde aus den Noten der Fächer Deutsch, Rechnen und Rechtschreiben in der 4 . Klasse Grundschule eine Durchschnittsnote gebildet. Die Noten der Fächer wurden von den Lehrern erfragt.

4 Die Studien sind unter den Nummern 0893 und $0894 \mathrm{im}$ Zentralarchiv für empirische Sozialforschung der Universität zu Köln archiviert. Die Untersuchungen wurden unter den Titeln ,Elternhaus und Bildungschancen" und „Lehrerurteil und Bildungschancen" durch die Arbeitsgruppe für empirische Bildungsforschung, Heidelberg, und das Institut für Demoskopie, Allensbach, durchgefuhrt. Projektleiter waren Rita Baur und Ruth Gresser-Spitzmüller.

5 Eine detaillierte Beschreibung der Variable findet sich in Wiese (1978).
Um den Einfluß der Lehrerempfehlung auf die Bildungsentscheidung der Eltern feststellen zu können, müssen die Bildungspräferenzen der Eltern bekannt sein, die diese hatten, bevor der Lehrer seine Empfehlung ausgesprochen hat. In der Befragung der Eltern sind Bildungspräferenzen der Eltern nicht erhoben worden. Hingegen sind die Lehrer nach schulischen Plänen der Eltern befragt worden. Ein Teil der Eltern hatte aber nicht mit den Lehrern gesprochen $(20 \%$ der ungewichteten Fälle). In diesen Fällen wurde nach den vom Lehrer vermuteten Plänen der Eltern gefragt. Von den Eltern wiederum, die mit dem Lehrer gesprochen hatten, hatte ein Teil lediglich vage Vorstellungen über die schulische Zukunft ihrer Kinder, ein anderer Teil konkrete Pläne. Aus diesen Gründen mußte die Variable Bildungspräferenz der Eltern aus mehreren Einzelfragen zusammengesetzt werden. Bei jeder Einzelfrage sind aber mehr oder weniger Ausfälle zu verzeichnen, d.h. die Lehrer konnten die schulischen Präferenzen der Eltern nicht angeben. Da Eltern höherer Schichtzugehörigkeit aber häufiger mit dem Lehrer gesprochen hatten und häufiger konkretere Pläne für ihre Kinder hatten, kam es zu einer schichtenspezifischen Verzerrung in der verbleibenden Stichprobe. Daraufhin wurden die Eltern, deren Bildungspräferenzen bekannt waren, erneut nach Merkmalen der Grundgesamtheit, nämlich der Berufsstellungsgruppe des Vaterberufs und dem Schultyp, umgewichtet. Tabelle 1 zeigt die Variable Bildungspräferenz der Eltern, deren Zusammensetzung aus den Einzelfragen und die Häufigkeiten nach der Umgewichtung.

Uber die Gültigkeit der Lehrerangaben können keine Aussagen gemacht werden. Wenn man annimmt, daß die Lehrer die Angaben teilweise aufgrund vorgefaßter Meinungen über den $\mathrm{Zu}$ sammenhang zwischen Elternstatus und Bildungslaufbahn des Kindes gemacht haben - was besonders im Falle der vermuteten Präferenzen plausibel ist -, dann sind die Bildungspräferenzen der Eltern systematisch verzerrt. Dies würde zu einer Erhöhung der Korrelation zwischen Status und Bildungspräferenzen der Eltern führen und nicht nur die Elternpräferenzen stärker status- und weniger leistungsbezogen erscheinen lassen als sie tatsächlich sind, sondern könnte auch zu einer Unterschätzung der Einflußstärke der Elternpräferenz bzw. einer Uberschätzung der Einflußstärke der Lehrerempfehlung auf die Bil- 
TABELLE 1 Bildungspräferenzen der Eltern (gewichtet für $N=959$ )

\begin{tabular}{ll}
\hline Fragen an den Lehrer & $\begin{array}{l}\text { Bildungspräferenzen der Eltern } \\
\text { schule schule sium }\end{array}$ \\
\hline
\end{tabular}

Haben die Eltern von

(Name des Kindes)

mit Ihnen dariber ge-

sprochen, auf welche

Schule sie ihr Kind

schicken sollen?

- wenn ja:

Hatten da die Eltern

schon bestimmte Vor-

stellungen, was mit

dem Kind nach der 4.

Klasse geschehen soll-

te, oder war es noch

ganz offen?

- bestimmte Vor-

stellungen

$139 \quad 179 \quad 205$

523

- noch ganz offen:

Hatten Sie den Ein-

druck, daß die El-

tern doch eine be-

stimmte Vorliebe

für einen Weg hatten?

\section{- ja}

- wenn nein:

Hatten Sie eine Vermutung, welche Pläne die Eltern mit ihrem

Kind nach der

4. Klasse hatten?

\begin{tabular}{lcccc}
- ja & 233 & 22 & 31 & 286 \\
\hline Insgesamt & 402 & 286 & 271 & 959 \\
$\%$ & 41.9 & 29.8 & 28.2 & $100 \%$ \\
\hline
\end{tabular}

dungsentscheidung führen. Zweifel an der Gültigkeit der Lehrerangaben können im Rahmen der vorliegenden Untersuchung nicht ausgeräumt werden. Wären die Bildungspräferenzen von den Eltern selbst erfragt worden, so hätte die Gefahr bestanden, daB die Eltern ihre Präferenzen den bereits getroffenen Bildungsentscheidungen ange$\mathrm{paßt}$ hätten. Eine longitudinale Vorgehensweise bei der Primäruntersuchung wäre methodisch angemessen gewesen.

\section{Ergebnisse}

Die zu den verschiedenen Fragestellungen abgeleiteten Hypothesen können jetzt den empiri- schen Ergebnissen der multivariaten Kausanalyse gegenübergestellt werden. In Abbildung 5 und Tabelle 2 sind die Ergebnisse der Pfadanalyse dargestellt. Tabelle 2 unterteilt die Kausalstruktur des Gesamtmodells in einzelne Teilmodelle entsprechend den Fragestellungen und Hypothesen im theoretischen Teil der Arbeit.

Zunächst werden die Teilmodelle „Elternpräferenz", „,Lehrerempfehlung“ und „Bildungsentscheidung", danach Status- und Leistungseffekte behandelt, die sich im „Gesamtmodell" ergeben. Anschließend folgen weitergehende Uberlegungen zum direkten und indirekten Schichteffekt auf die Empfehlung des Lehrers.

\subsection{Die Bildungspråferenzen der Eltern}

Die Bildungswünsche der Eltern wurden als stark durch statusbedingte Interessen und Ansprüche bestimmt angesehen. Die schulischen Leistungen des Kindes können von den Eltern zwar nicht völlig ignoriert werden, sie sind aber vergleichsweise von geringerer Bedeutung. Entsprechend wurde ein direkter Schichteffekt auf die Präferenzen der Eltern angenommen, der stärker ist als der indirekte, über die Schulleistung vermittelte Schichteffekte. Beide Hypothesen werden durch die empirischen Ergebnisse bestätigt (siehe Teilmodell „Elternpräferenz" in Tabelle 2). Die schulischen Präferenzen der Eltern sind stärker status- als leistungsbezogen (Pfad PREST, ELPRAEF $=.427 \mathrm{zu}$ Pfad DNOTE, ELPRAEF = .377), der direkte Schichteffekt ist stärker als der indirekte Schichteffekt (Pfad PREST, ELPRAEF $=.427 \mathrm{zu}$ Pfad PREST, DNOTE, ELPRAEF $=.103)$.

\subsection{Die Empfehlungen der Lehrer}

Im Gegensatz zu den Eltern wurde den Lehrern kein unmittelbares Interesse an einer statusbezo-

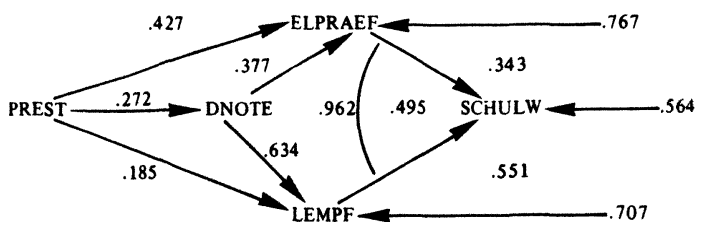

ABBILDUNG 5 Gesamtmodell $N=758$, listwise deletion (vgl. Tabelle 2) 
TABELLE 2 Gesamtmodell $\mathrm{N}=758^{*}$

\begin{tabular}{|c|c|c|c|c|c|c|c|}
\hline \multicolumn{5}{|c|}{ Kausaler Effekt } & $\begin{array}{l}\text { Nicht- } \\
\text { kausaler } \\
\text { Effekt }^{+}\end{array}$ & $\begin{array}{l}\text { Rest der } \\
\text { Test- } \\
\text { korrelation } \S\end{array}$ & $\begin{array}{l}\text { Erklärte } \\
\text { Varianz }\end{array}$ \\
\hline & (1) & (2) & (3) & (4) & (5) & (6) & (7) \\
\hline \multicolumn{8}{|c|}{ Teilmodell Elternpräferenz } \\
\hline $\begin{array}{l}\text { PREST, ELPRAEF } \\
\text { PREST, DNOTE } \\
\text { DNOTE, ELPRAEF }\end{array}$ & $\begin{array}{l}.530 \\
.272 \\
.493\end{array}$ & $\begin{array}{l}.427 \\
.272 \\
.377\end{array}$ & $\begin{array}{c}.103 \text { über DNOTE } \\
- \\
-\end{array}$ & $\begin{array}{l}.530 \\
.272 \\
.377\end{array}$ & $\begin{array}{l}- \\
- \\
.116 \text { vor } \\
\text { PRI }\end{array}$ & $\mathrm{EST}^{-}$ & $\begin{array}{c}.412 \\
.074 \\
-\end{array}$ \\
\hline \multicolumn{8}{|c|}{ Teilmodell Lehrerempfehlung } \\
\hline $\begin{array}{l}\text { PREST, LEMPF } \\
\text { PREST, DNOTE } \\
\text { DNOTE, LEMPF }\end{array}$ & $\begin{array}{l}.357 \\
.272 \\
.684\end{array}$ & $\begin{array}{l}.185 \\
.272 \\
.634\end{array}$ & $\begin{array}{l}.172 \text { über DNOTE } \\
- \\
-\end{array}$ & $\begin{array}{l}.357 \\
.272 \\
.634\end{array}$ & $\begin{array}{l}. \\
.050 \text { von } \\
\text { PRI }\end{array}$ & $\begin{array}{l}- \\
n \\
\text { n } \\
\text { EST }\end{array}$ & $\begin{array}{c}.500 \\
.074 \\
-\end{array}$ \\
\hline
\end{tabular}

Teilmodell Bildungsentscheidung

\begin{tabular}{|c|c|c|c|c|c|c|c|}
\hline \multirow[t]{2}{*}{ ELPRAEF, SCHULW } & \multirow[t]{2}{*}{.721} & \multirow[t]{2}{*}{.343} & \multirow[t]{4}{*}{ - } & \multirow[t]{2}{*}{.343} & \multicolumn{3}{|c|}{$\begin{array}{l}.273 \text { von LEMPF } \\
.104 \text { von PREST }\end{array}$} \\
\hline & & & & & .377 & - & - \\
\hline \multirow[t]{2}{*}{ LEMPF, SCHULW } & .787 & .551 & & .551 & \multicolumn{3}{|c|}{$\begin{array}{l}.170 \text { von ELPRAEF } \\
.065 \text { von PREST }\end{array}$} \\
\hline & & & & & .235 & - & - \\
\hline \multirow[t]{2}{*}{ ELPRAEF, LEMPF } & .684 & - & - & - & \multicolumn{3}{|c|}{$\begin{array}{l}.495 \text { Korrelation } d . \\
\text { Residuen } \\
.189 \text { von PREST }\end{array}$} \\
\hline & & & & & .684 & - & - \\
\hline \multicolumn{8}{|c|}{ Gesamtmodell } \\
\hline \multirow[t]{3}{*}{ PREST, SCHULW } & .356 & - & \multicolumn{2}{|c|}{$\begin{array}{l}.146 \text { über ELPRAEF } \\
.035 \text { über DNOTE/ELPRAEF }\end{array}$} & & & \\
\hline & & & \multicolumn{2}{|c|}{$\begin{array}{l}.102 \text { über LEMPF } \\
.095 \text { über DNOTE/LEMPF }\end{array}$} & & & \\
\hline & & & .378 & .378 & - & -.022 & .681 \\
\hline \multirow[t]{3}{*}{ DNOTE, SCHULW } & .562 & - & $\begin{array}{l}.129 \text { über ELPRAEF } \\
.349 \text { über LEMPF }\end{array}$ & & \multicolumn{2}{|c|}{$\begin{array}{c}.040 \text { von PREST/ } \\
\text { ELPRAEF }\end{array}$} & \\
\hline & & & .478 & .478 & \multicolumn{2}{|c|}{$\begin{array}{c}.028 \text { von PREST/ } \\
\text { LEMPF } \\
.010 \text { von ELPRAEF/ } \\
\text { LEMPF } \\
.011 \text { von LEMPF/ } \\
\text { ELPRAEF }\end{array}$} & \\
\hline & & & & & .089 & -.005 & - \\
\hline
\end{tabular}

* Durch fehlende Werte verringert sich die Anzahl der Fälle von $N=959$, Tabelle 1 , auf $N=758$

+ Effekte, die sich nicht aus einer Kausalkette gleicher, sondern wechselnder Richtungen ergeben. Opp und Schmidt (1976: 147 ff) unterscheiden Effekte von Drittvariablen und indirekt korrelierte Effekte. Effekte von Drittvariablen ergeben sich durch den Einfluß der Drittvariable auf zwei miteinander korrelierende Variable, indirekt korrelierte Effekte aufgrund der Korrelation zwischen zwei Variablen, die aus theoretischen Gründen nicht als kausal miteinander verbunden angenommen werden.

§ Der Rest der Testkorrelation bei überidentifizierten Modellen ist ein Gütemaß für das aufgestellte Modell. Bei gerade identifizierten Kausalmodellen liegen genauso viele Pfade wie Korrelationen vor. Werden Pfade aus theoretischen Gründen eleminiert, so liegt ein überidentifiziertes Modell vor. 
genen Auslese unterstellt. Als gültiges Handlungsprinzip des Lehrers wurde das Leistungsprinzip angesehen. Schulische Leistungskriterien sind die wesentliche Orientierungsgröße der Empfehlung. Bedingungen, die mit der sozialen Herkunft des Schülers zusammenhängen, können aber zu einem - allerdings vergleichsweise geringen - Schichteffekt auf die Lehrerempfehlung führen. Der indirekte Schichteffekt, der über schichtenabhängig ungleiche Schulnoten vermittelt wird, wurde als geringer im Vergleich zum direkten Schichteffekt veranschlagt, weil nur ein geringer Einfluß der Schichtzugehörigkeit auf die Schulnoten des Schülers angenommen wurde. Die Hypothesen werden ebenfalls durch die empirischen Ergebnisse bestätigt (siehe Teilmodell „Lehrerempfehlung" in Tabelle 2). Die wesentliche Orientierungsgröße der Lehrerempfehlung sind die Schulnoten, statusbedingte Einfliusse haben vergleichsweise geringe Bedeutung (Pfad DNOTE, LEMPF $=.634 \mathrm{zu}$ Pfad PREST, LEMPF $=.185)^{6}$. Der direkte Schichteffekt ist beinahe gleich stark wie der indirekte Schichteffekt (Pfad PREST, LEMPF $=.185 \mathrm{zu}$ Pfad PREST, DNOTE, LEMPF =

.172). Der Sachverhalt, daß Lehrer häufiger Kinder höherer Schichtzugehörigkeit auf die höheren weiterführenden Schulen empfehlen, ist demnach einmal darauf zurückzuführen, daß durch die Schichtzugehörigkeit des Kindes die Schulnoten beeinflußt sind und die Empfehlung des Lehrers stark notenbezogen ist. Dieser indirekte Schichteffekt auf die Lehrerempfehlung ergibt sich zwangsläufig und ist eine vom Lehrer unbeeinflußbare Konsequenz der starken Orientierung an schulischen Leistungsmaßstäben. Der direkte Schichteffekt auf die Übergangsempfehlung dage-

6 Um den formal-statistischen Anforderungen der $P$ fadanalyse annähernd zu genügen, wurde als schulisches Leistungskriterium die Durchschnittsnote aus den Noten der Fächer Deutsch, Rechnen und Rechtschreiben der 4 . Klasse Grundschule gebildet. Mit der Durchschnittsnote als Leistungskriterium wird aber die Leistungsbezogenheit der Lehrerempfehlung unterschätzt. Grundlage der Lehrerempfehlung sind bestimmte Schwellenwerte in den Zensuren der Hauptfächer, also etwa Noten besser/schlechter als 3.0. Unter der Annahme, daß sich alle Lehrer an diesen Schwellenwerten orientieren, ergibt sich eine vollkommende Korrelation zwischen Schulleistung (dichotomisiert gute/schlechte Noten) und Lehrerempfehlung (dichotomisiert Volksschule/Realschule und Gymnasium). Unter der gleichen Annahme ist bei Verwendung der Durchschnittsnote als Leistungskriterium eine vollkommene Korrelation mit der Lehrerempfehlung wegen des größeren Wertebereichs nicht möglich. gen liegt im Einflußbereich und in der Verantwortung des Lehrers, wobei zunächst offen ist, wie dieser Effekt interpretiert und damit bewertet wird. Er wurde hier damit erklärt, daß Lehrer ihre Empfehlungen neben den Schulnoten auch an sozialen Bedingungen des Elternhauses orientieren. Allerdings ist diese These durch die Pfadanalyse noch nicht belegt. Der direkte Schichteffekt auf die Lehrerempfehlung kann ebenso damit erklärt werden, daß Lehrer Interesse an der Stabilisierung der Sozialstruktur haben und deshalb soziale Aufstiege von Unterschichtmitgliedern weniger häufig zulassen. Andere Erklärungsversuche des direkten Schichteffekts werden später noch behandelt werden.

\subsection{Die Bildungsentscheidung der Eltern}

Das dritte Teilmodell bezog sich auf die Bedeutung von Elternpräferenz und Lehrerempfehlung für die Bildungsentscheidung, also den tbergang des Schülers auf eine der weiterführenden Schulen. Die Hypothese, daß der Einfluß der Elternpräferenz stärker ist als der Einfluß der Lehrerempfehlung wurde damit begründet, da $B$ die Eltern ihre statusbezogenen Interessen durchsetzen wollen. Schon die schulischen Präferenzen der Eltern wurden als vergleichsweise schwach leistungsbezogen angenommen, entsprechend wurde eine schwächere Orientierung der Eltern an den stärker leistungsbezogenen Lehrerempfehlungen angenommen. Hier unterscheidet sich das empirische Ergebnis von der Hypothese (siehe Teilmodell „Bildungsentscheidung“ in Tabelle 2). Die Bildungsentscheidungen der Eltern werden stärker von den Empfehlungen der Lehrer beeinflußt (Pfad LEMPF, SCHULW $=.551 \mathrm{zu}$ Pfad ELPRAEF, SCHULW = .343). Ein Teil der Eltern setzt nicht die ursprünglichen schulischen Pläne und Wünsche durch, sondern orientiert sich bei der endgültigen Entscheidung der Schullaufbahn an den Empfehlungen der Lehrer.

\subsection{Status- und Leistungseffekt auf die Bildungsentscheidung}

Bevor die Ergebnisse des Gesamtmodells behandelt werden, soll kurz auf dessen Kausalstruktur eingegangen werden. Das vorliegende Modell (Gesamtmodell in Tabelle 2 und Abbildung 5) wurde dadurch begründet, daB mit den Bildungsvorstellungen der Eltern und der Lehrer die rele- 
vanten Einflußgrößen auf die Bildungsentscheidung erfaßt werden und mit der Schichtzugehörigkeit der Eltern und den Schulleistungen der Kinder die relevanten Bestimmungsfaktoren für die Bildungsvorstellungen von Eltern und Lehrern. Deshalb wurden eigenständige, direkte Kausaleinfluisse des Elternstatus und der Schulleistungen des Kindes auf die Bildungsentscheidung nicht zugelassen. Der Test des Gesamtmodells bestätigt die vermutete Kausalstruktur ${ }^{7}$.

Die Fragestellung der Untersuchung, welche Bedeutung die Lehrerempfehlung letztlich für die schichtenspezifische Auslese am Ende des 4. Schuljahres hat, kann erst durch die Kausalbeziehungen des Gesamtmodells, durch das Zusammenwirken aller Variablen, beantwortet werden. Bei der Aufstellung der Hypothesen wurde der Einfluß der Lehrerempfehlung auf die Bildungsentscheidung der Eltern unterschätzt. Die starke Orientierung der Eltern an der Lehrerempfehlung hat aber Auswirkungen bei der Vermittlung von Schicht- und Leistungseffekten auf die Bildungsentscheidung. Aufgrund der Hypothesen mußte ein starker Einfluß des Elternstatus über die Bildungspräferenzen der Eltern angenommen werden und eine relativ geringe Bedeutung der stärker leistungsorientierten Lehrerempfehlung. Nach den empirischen Ergebnissen haben aber die Empfehlungen der Lehrer starken Einfluß auf die Entscheidung der Schullaufbahn, und damit verändern sich auch die Schicht- und Leistungseffekte auf die Bildungsentscheidung. Da die Lehrerempfehlung vergleichsweise stärker leistungsorientiert ist, wird der Einfluß der stärker statusbezogenen Elternpräferenzen zugunsten einer leistungsorientierten Auslese zurückgedrängt. Obwohl die Lehrerempfehlungen selbst nicht von Schichteinfliissen frei sind und auch nicht völlig frei sein können, da die Schulleistung des Kindes mit dem Elternstatus korreliert, haben sie letztlich einen nivellierenden Einfluß auf die Ungleichheit der schulischen Bildungslaufbahnen, wenn man die schulischen Leistungen der Kinder berücksichtigt.

7 Die Kausalstruktur eines Modells wird getestet, indem alternative Modelle, deren Kausalstruktur von der theoretisch angenommenen abweicht, gerechnet werden. Pfade, deren standardisierter, partialisierter Regressionskoeffizient kleiner als .10 ist, werden nach den Konventionen der Pfadanalyse aus dem Modell gestrichen, d.h. der Einfluß wird als unbedeutend angesehen.
Im einzelnen können folgende Schichteffekte auf die Bildungsentscheidung unterschieden werden (siehe Gesamtmodell in Tabelle 2): Der gesamte Schichteffekt auf die Bildungsentscheidung (Pfad PREST, SCHULW = .356) wird ungefähr je zur Hälfte über die Elternpräferenz (indirekt vermittelte Kausaleffekte über ELPRAEF und DNOTE/ELPRAEF $=.181$ ) und über die Lehrerempfehlung (indirekt vermittelte Kausaleffekte über LEMPF und DNOTE/LEMPF $=.197$ ) vermittelt. Der Schichteffekt auf die Lehrerempfehlung ist zwar im Vergleich zum Schichteffekt auf die Eltempräferenz schwächer, er gewinnt aber an Bedeutung durch den starken Einfluß der Lehrerempfehlung auf die Bildungsentscheidung. Beides, Lehrerempfehlung und $\mathrm{El}$ ternpräferenz, trägt also in beinahe gleichem Maße zu sozial ungleichen Bildungslaufbahnen der Schüler bei. Während aber der über die Lehrerempfehlung vermittelte Schichteffekt wiederum zu $48 \%$ über schulische Leistungen der Kinder vermittelt ist (indirekt vermittelter Kausaleffekt über LEMPF $=.102 \mathrm{zu}$ indirekt vermitteltem Kausaleffekt über DNOTE/LEMPF $=.095$ ), ist der über die Elternpräferenz vermittelte Schichteffekt nur zu 19\% über Schulleistungen der Kinder vermittelt (indirekt vermittelter Kausaleffekt über ELPRAEF $=.146 \mathrm{zu}$ indirekt vermitteltem Kausaleffekt über DNOTE/ELPRAEF $=.035$ ). Während also die Uberrepräsentation von Kindern höherer Schichtzugehörigkeit, die durch die Lehrerempfehlungen zustandekommt, in stärkerem Maße nach Leistungsgesichtspunkten gerechtfertigt ist, ist der Schichteffekt, der über die Elternpräferenz auf die Bildungsentscheidung vermittelt wird, fast vollständig auf statusbezogene Interessen zurückzuführen. Damit tragen die Empfehlungen der Lehrer, obwohl selbst nicht von Schichteinflüssen frei, letztlich zu einer stärker leistungsorientierten Auslese bei. Die volle Bedeutung der Lehrerempfehlung aber wird erst deutlich, wenn hypothetisch gefragt wird, welche Konsequenzen ein Fehlen der Lehrerempfehlungen hätte. Die Bildungspräferenz der Eltern entspräche der Bildungsentscheidung und die schichtspezifische Auslese würde sich erhöhen $(\mathrm{r}$ PREST, ELPRAEF $=.530$ ).

\subsection{Weitergehende Überlegungen zum direkten und indirekten Schichteffekt auf die Lehrer- empfehlung}

Auch wenn die Empfehlungen der Grundschullehrer letztlich einen nivellierenden Einfluß auf 
die Bildungsentscheidungen der Eltern haben, so bleibt dennoch der nicht durch schichtabhängig ungleiche Schulleistungen der Kinder erklärbare direkte Schichteffekt auf die Lehrerempfehlung bestehen. Die Untersuchungen, die sich mit den Bestimmungsgründen der Lehre rempfehlung beschäftigen, kommen zu keinen schlitssigen Ergebnissen, was die Ursachen des direkten Schichteffekts angeht. Steinkamp (1967: 318) führt die Abweichung der Lehrerempfehlung von der meß. baren Leistung auf die Berücksichtigung zusätzlicher schichtenspezifisch verteilter, subjektiver und leistungsfremder Kriterien der Lehrerempfehlung zurück. Zusätzlich zu guten Noten geforderte Leistungs- und Verhaltensmerkmale des Schülers haben einen diskriminierenden Effekt für die Kinder unterer Schichten. Allerdings belegt Steinkamp diese These nur indirekt. Lehrer geben explizit $z u$, neben zufriedenstellenden Leistungen des Kindes noch andere Kriterien für ihre Empfehlung zu berücksichtigen (Steinkamp 1967: 306), und diese Kriterien entsprechen weitgehend dem Erziehungsideal der Lehrer (Steinkamp 1967: 313). Die Verteilung dieser zusätzlich geforderten Qualitäten nach dem Urteil der Lehrer zeigt, daß Kinder aus oberen Schichten diese Eigenschaften fast doppelt so häufig aufweisen (Steinkamp 1967: 313). Die tatsächliche Handlungsrelevanz dieser Zusatzkriterien für die Empfehlung wird jedoch nicht nachgewiesen. Die Untersuchungen von Preuß (1968: 146) und Gresser-Spitzmüller (1973: 145) belegen, daß selbst gute Noten und positive charakterologische Beurteilung von Unterschichtkindern nicht deren Chancen verbessern, auf höhere Schulen empfohlen zu werden. Die These, daß die diskriminierende Wirkung der Lehrerempfehlung durch die Verwendung von Zusatzkriterien zustandekommt, ist nicht eindeutig belegt.

Die These der Mittelschichtgebundenheit der Lehrer erklärt den Schichteinfluß auf die Lehrerempfehlung damit, daß Lehrer die Schüler nach ihren eigenen mittelschichtspezifischen Leistungsund Verhaltensstandards beurteilen. Dabei können Handlungsmotive des Lehrers wirksam werden, die mit einem Interesse an der Stabilisierung der Sozialstruktur zusammenhängen. Soziale Aufstiege von Unterschichtmitgliedern werden nicht zugelassen, da dadurch die eigene soziale Plazierung gefährdet ist. Aber auch diese These wird durch die empirischen Ergebnisse nicht gestützt. Preuß (1968: 154), Steinkamp (1967:
320) und Gresser-Spitzmüller (1973: 193) untersuchen den Einfluß der sozialen Herkunft des Lehrers auf die Empfehlung. Die sehr schwachen Beziehungen und die teilweise widersprüchlichen Ergebnisse bestätigen die These der Mittelschichtgebundenheit des Grundschullehrers nicht.

Weiter oben sind noch andere Erklärungen zum Schichteinfluß auf die Lehrerempfehlung dargelegt worden. Neben der Erklärung, daß bessere Kontakte zwischen Eltern höherer Schichtzugehörigkeit und Lehrern einen Einfluß haben, scheint vor allem plausibel, daß Lehrer bei ihren Empfehlungen die sozialen Bedingungen des Elternhauses berücksichtigen. Lehrer geben ihre Empfehlung aufgrund der Risiken und Chancen, die mit einer höheren Schullaufbahn verbunden sind und die sich mit der sozialen Herkunft des Schülers verändern. Schüler, deren schulische Leistungen zufriedenstellend sind, die aber durch das Elternhaus emotional und intellektuell nicht unterstützt werden und deren Durchhaltevermögen dadurch von vornherein fraglich ist, sollen vor Mißerfolg bewahrt werden. Anhand der Variablen der vorliegenden Untersuchungen läßt sich diese These jedoch nicht überprüfen. Die Untersuchungen, die sich mit den Bestimmungsgründen der Lehrerempfehlung beschäftigen, scheinen eher daraufhin angelegt zu sein, soziale Vorurteile von Lehrern aufzudecken.

Keine der theoretischen Erklärungen zum direkten Schichteffekt ist also empirisch abgesichert. Durch welche Gründe und Motive des Lehrers er auch immer bedingt ist, er liegt im Handlungs- und Einflußbereich des Lehrers. Damit ist noch nicht gesagt, daß er nicht vom Lehrer verantwortet werden kann, also auf illegitime Diskriminierungen von Unterschichtkindern zurückzuführen ist.

Dagegen ergibt sich der indirekte Schichteffekt nicht durch die Berücksichtigung von Empfehlungskriterien, die mit der sozialen Herkunft des Schülers zusammenhängen, sondern durch das Zusammenwirken von schichtspezifisch ungleichen Noten und beruflichen Handlungsnormen des Lehrers. Je stärker der Lehrer seine Empfehlung an schulischen Leistungskriterien orientiert, um so stärker wird - bei gegebenem Einfluß der Schichtzugehörigkeit auf die Schulnoten des Schülers - der indirekte Schichteffekt auf die Lehrerempfehlung. Aus der Sicht des 
Lehrers sind Schulnoten valide Indikatoren der schulischen Leistungsfähigkeit des Schülers, an denen er seine Empfehlung im wesentlichen orientieren muß. Der indirekte Schichteffekt liegt deshalb bei gegebenen Noten nicht in seinem Einfluß- und Handlungsbereich ${ }^{8}$. Er ergibt sich aus dem Zusammenwirken von Folgen der Sozialstruktur und beruflichen Handlungsnormen des Lehrers.

Allerdings kann man vermuten, daß der Lehrer schon bei der Notengebung seine Einflußmög. lichkeiten wahrnimmt. Der Schichteinfluß auf die Durchschnittsnote muß nicht ausschließlich auf das Wirken sozialstruktureller Vor- und Nachteile von Schülern zurückzuführen sein, sondern kann auch dadurch zustandekommen, daß der Lehrer Kriterien der Leistungsbeurteilung berücksichtigt, nach denen Unterschichtkinder schlechter abschneiden als Oberschichtkinder. Kriterien wie Motivation, Disziplin und sprachliche Ausdrucksfähigkeit des Schülers, aber auch soziale Vorurteile des Lehrers gegenüber Unterschichtkindern können zu einer Unterbewertung der Schulleistungen bei Unterschichtkindern und zu einer Überbewertung bei Oberschichtkindern führen.

Welche Konsequenzen ergeben sich hieraus für die Interpretation des indirekten Schichteffekts auf die Lehrerempfehlung? Können aus der Stabilisierung schichtabhängiger Leistungsunterschiede Handlungsmotive des Lehrers abgeleitet werden, die bei der Empfehlung wirksam werden? Eine solche Verbindung der Handlungsmotive bei der Notengebung und der Empfehlung würde zu folgender Interpretation des indirekten Schichteffekts auf die Empfehlung führen: Bei der Notengebung diskriminiert der Lehrer Unterschichtkinder durch die Unterbewertung ihrer Leistungen und läßt diese Diskriminierung bei scheinbarer Leistungsgerechtigkeit in der Empfehlung wirksam werden. Gegen eine solche Interpretation sprechen verschiedene Gründe: Einmal ist das Handeln des Lehrers weitgehend an Leistungsmerkmalen und weniger an Statusmerkmalen des Schülers orientiert. Daß das Leistungsprinzip als Handlungsprinzip weitgehend guiltig ist, zeigt sich bei der Empfehlung. Der Einfluß

8 Jede Ungleichbehandlung von Schülern mit gleicher Durchschnittsnote - welche Motive und Überlegungen des Lehrers auch immer dahinterstehen - zeigt sich im direkten Schichteffekt auf die Empfehlung. der Durchschnittsnote auf die Lehrerempfehlung ist wesentlich stärker als der Einfluß des Elternstatus (siehe Abbildung 5, Tabelle 2: Pfad DNOTE/LEMPF $=.634 \mathrm{zu}$ Pfad PREST/LEMPF $=.185$ ). Zum zweiten kann nur ein Teil des Schichteffekts auf die Schulnoten (der relativ schwach ist: Pfad PREST, DNOTE = .272) auf nicht-strukturelle Bedingungen zurückgeführt werden, so daß die Einflußmöglichkeiten des Lehrers von vornherein begrenzt sind. Außerdem wird eine bewußte und planmäßige Unterbewertung der Schulleistungen von Unterschichtkindern und/oder Utberbewertung der Schulleistungen von Oberschichtkindern durch den Lehrer vorausgesetzt. Das Handeln des Lehrers aber bezieht sich auf den einzelnen Schüler, nicht auf Schichten oder die „Sozialstruktur“ einer Klasse.

Diese Gründe sprechen sowohl dagegen, daß der Lehrer sich eines von ihm ,produzierten“"

Schichteffekts auf die Schulnoten überhaupt bewußt ist, als auch gegen die Vorstellung, daß Handlungsmotive der Notengebung und der Empfehlung vom Lehrer in ihrer Wirkung aufeinander bezogen werden. Trotz einer möglichen Stabilisierung schichtenspezifischer Leistungsunterschiede durch den Lehrer ist deswegen die analytische Trennung des Schichteffekts auf die Lehrerempfehlung gerechtfertigt und die Deutung des direkten und indirekten Schichteffekts plausibel: Der direkte Schichteffekt auf die Lehrerempfehlung kommt dadurch zustande, daß der Lehrer Empfehlungskriterien zugrunde legt, die mit der sozialen Herkunft des Schülers zusammenhängen. Der indirekte Schichteffekt auf die Lehrerempfehlung dagegen ist Ergebnis des Strukt ureffekts auf die schulische Leistungsfähigkeit des Kindes - gemessen an schulischen Leistungskriterien und der Verbindlichkeit des Leistungsprinzips für den Lehrer. Der direkte Schichteffekt liegt im Handlungs- und Verantwortungsbereich des Lehrers, den indirekten Schichteffekt dagegen kann er aufgrund seiner beruflichen Handlungsnormen nicht beeinflussen. Er ergibt sich zwangsläufig durch das Zusammenwirken des Schichteffekts auf die Schulnoten und die Leistungsorientierung des Lehrers.

\section{Schlußfolgerungen: Ein Dilemma der Noten- gebung in der Schule}

Indirekter und direkter Schichteffekt auf die Lehrerempfehlung und der Einfluß der Lehrer- 
empfehlung auf die Bildungsentscheidung dürfen aber nicht nur isoliert betrachtet, sondern müssen im Zusammenhang gesehen werden. Faktisch überträgt - wie gezeigt - ein Teil der Eltern die Entscheidung auf die Lehrer, wenn es um die endgiiltige Entscheidung der Schullaufbahn des Kindes geht. Einerseits ist die Lehrerempfehlung stärker leistungsorientiert als die statusbezogenen Präferenzen der Eltern und hat dadurch nivellierenden Einfluß auf die Bildungsentscheidung, andererseits diskriminiert sie - aus welchen Gründen auch immer - Kinder unterer Schichten.

Ob eine Objektivierung des Ausleseverfahrens durch einen Schulleistungstest sinnvoll ist, hängt wesentlich davon ab, wie der direkte Schichteinfluß auf die Lehrerempfehlung interpretiert wird. Ist der direkte Schichteffekt darauf zurückzuführen, daß der Lehrer wegen seiner Mittelschichtgebundenheit die eigenen Leistungs- und Verhaltensstandards zugrundelegt und damit Unterschichtkinder benachteiligt, dann ist eine Objektivierung der Auslese wünschenswert. Welche Schullaufbahn ein Schüler einschlägt, soll nicht von der „Lehrerpersönlichkeit" und individuellen Vorstellungen der Lehrer über Leistungsfähigkeit und Verhaltensstandards von Schülern abhängen. Kann der direkte Schichteffekt auf die Lehrerempfehlung allerdings damit erklärt werden, daß der Lehrer in seiner Empfehlung die Erfolgschancen des Schülers in Abhängigkeit von den sozialen Bedingungen berücksichtigt, dann ist die Forderung nach einer Objektivierung der Auslese anders zu beurteilen. Der direkte Schichteinfluß auf die Lehrerempfehlung ist dann unabhängig von individuellen Vorstellungen der Lehrer. Vielmehr stellt der Lehrer seine eigenen persönlichen Vorstellungen zurück und kalkuliert die Chancen des Schülers aus dessen Sicht. Die auf den einzelnen Schüler bezogene individuelle Erfolgsprognose scheint zuverlässiger als die Prognose aufgrund gemessener Leistung, weil in ihr soziale Bedingungen des Elternhauses, Motivation und Durchhaltevermögen des einzelnen Schülers berücksichtigt werden kann. Eine ausschließlich schulnotenbezogene Auslese könnte zu häufigerem Scheitern von Schülern unter Sozialschichten in höheren Bildungsgängen führen.

Weiter könnte das Ersetzen der Lehrerempfehlung durch einen Schulleistungstest dazu führen, daß sich letztlich stärkere Schichteffekte auf die Bildungsentscheidungen durchsetzen als bei Empfehlungen der Grundschullehrer, nämlich dann, wenn sich die Bildungsentscheidungen der Eltern ebensowenig an den Testergebnissen orientieren, wie die schulischen Präferenzen der Eltern an den Schulnoten orientiert sind. Das Urteil des Lehrers - in den Augen der Eltern der Fachmann für Erziehung und Bildung - hat größeres Gewicht als die gemessene Leistung des Kindes. Eine Ergänzung der Lehrerempfehlung durch einen Schulleistungstest scheint daher eine angemessene Lösung zu sein. Einerseits entstünde dadurch ein Druck auf die Lehrer, ihre Empfehlungen noch stärker an Leistungskriterien $\mathrm{zu}$ orientieren, andererseits ginge der Einfluß des Lehrers als Vermittler leistungsbezogener Selektionskriterien mit seiner nivellierenden Wirkung auf die Bildungsentscheidungen der Eltern nicht verloren.

\section{Literatur:}

Baur, Rita, 1972: Elternhaus und Bildungschancen. Eine Untersuchung über die Bedeutung des Elternhauses für die Schulwahl nach der 4. Klasse der Grundschule. Weinheim: Beltz.

Baur, Rita/Jaeger, A./Schönfelder, W., 1970: Zur Genauigkeit der Angaben über die soziale Herkunft der Schüler. Arbeitsgruppe für Empirische Bildungsforschung, Diskussionsbeiträge, Heft 2. Heidelberg.

Gordon, Robert A., 1968: Issues in Multiple Regression. The American Journal of Sociology 73.

Gresser-Spitzmüller, Ruth, 1973: Lehrerurteil und Bildungschancen. Eine Untersuchung über den Einfluß des Grundschullehrers auf die Wahl der weiterführenden Schulen. Weinheim, Basel: Beltz.

Kleining, G./Moore, H., 1968: Soziale Selbsteinstufung. Kölner Zeitschrift für Soziologie und Sozialpsychologie 20: 502-552.

Li, Ching-Chun, 1975: Path Analysis - a Primer. The Boxwood Press.

Meulemann, H., 1979: Soziale Herkunft und Schullaufbahn. Arbeitsbücher zur sozialwissenschaftlichen Methodenlehre. Frankfurt/New York: Campus.

Meulemann, H., 1979: Klassenlage, Entscheidungsfeld und Bildungsaspirationen. Ein Versuch zur theoretischen Präzisierung und kausalen Erklärung von Zusammenhängen zwischen sozialer Struktur und individueller Lebensplanung. Zeitschrift für Soziologie 8: $391-414$.

Opp, K.D./Schmidt, P., 1976: Einführung in die Mehrvariablenanalyse. Reinbek bei Hamburg: rororoStudium. 
Preuß, Otmar, 1968: Soziale Determinanten im Urteil von Volksschullehrern über die Eignung von Schülern für weiterführende Schulen. Unveröffentlichte Dissertation.

Steinkamp, Günther, 1967: Die Rolle des Volksschullehrers im schulischen Selektionsprozeß. Hamburger Jahrbuch für Wirtschafts- und Gesellschaftspolitik.
Wiese, W., 1978: Elternhaus und schulische Aspirationen. Eine sekundäranaly tische Untersuchung des Einflusses von Klassenlage, Arbeitserfahrungen und familiärer Umwelt auf die Entscheidung der Eltern über die weitere Schullaufbahn des Kindes am Ende des 4. Schuljahres. Diplomarbeit im Fach Soziologie, Köln. 